\title{
Simulations of observations with the Optical Monitor of the X-ray Multi-Mirror Satellite
}

\author{
P. Royer ${ }^{\star}$, J. Manfroid ${ }^{\star \star}$, E. Gosset ${ }^{\star \star \star}$, and J.-M. Vreux \\ Institut d'Astrophysique, Université de Liège, 5 avenue de Cointe, B-4000 Liège, Belgium
}

Received January 21; accepted May 19, 2000

\begin{abstract}
This paper addresses the question of the observations to be performed with the Optical Monitor (OM) of the X-ray Multi-Mirror Satellite (XMM) under several aspects. First, we discuss XMM-OM's photometric system and its colour transformations towards the standard $U B V$ system. Second, we establish a set of procedures to determine the temperature and the amount of interstellar absorption affecting the observed stars. Last, we address the possibility of isolating quasars in multidimensional colour diagrams based on the XMM-OM filter set.
\end{abstract}

Key words: satellite: XMM — photometry: colour transformations; interstellar absorption; quasars: identification of candidates

\section{Introduction}

Thanks to its unprecedented large X-ray collecting power, the XMM satellite (for X-ray Multi-Mirror) is expected to discover a wealth of new X-ray sources. In order to allow a quick and reliable identification of the sources, as well as to enable multiwavelength monitoring of their variability, the satellite is equipped with an optical complement known as the optical monitor, XMM-OM. This particular element is co-aligned with the three X-ray telescopes and consists of a $30 \mathrm{~cm}$ Ritchey-Chrétien telescope coupled with a photoncounting detector. The latter consists of a photocathode followed by three micro-channel plates as intensifier and by a tapered fiber-optic bundle connected to a fast scanning CCD. The usable area is made of 256 by 256 physical

Send offprint requests to: P. Royer, e-mail: proyer@ulg.ac.be

* Aspirant au Fonds National de la Recherche Scientifique, Belgium.

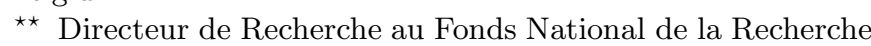
Scientifique, Belgium.

$\star \star \star$ Chercheur Qualifié au Fonds National de la Recherche Scientifique, Belgium. pixels but a centroiding process locates the events to $1 / 8$ of a pixel thus mimicking a 2048 by 2048 device. The field of view is 17 arcmin by 17 arcmin with a centroided pixel size of 0.5 arcsec. At the end of the OM exposure, the cumulated image is downloaded to the ground. An engineering mode allows to transmit the whole image but the routine science mode necessitates predefined windowing and/or binning. The telescope was designed so that the limiting magnitude would be no less than 24 when working in unfiltered light. More details can be found in Fordham et al. (1992) and Mason et al. (1996).

XMM-OM holds a 6-filter, UV and optical, photometric system. Three bands of the system were designed to match the Johnson's UBV system (Johnson 1955; Bessel 1990). The rectangular profiles of the latter filters render the colour transformations between the XMM-OM and the Johnson systems quite complicated (see Royer \& Manfroid 1996 for a discussion on the untransformability of rectangularly shaped filters), or even not always possible. Such colour transformations will nevertheless remain necessary for those who will have to compare XMM-OM observations with ground-based ones. In this paper, we provide the reader with theoretical estimations of these colour transformations.

The XMM-OM filter set comprises three non-standard filters, exploring a wavelength domain (UV) where no extensive observations have been performed to date. Combined with the poor match between the XMM-OM optical filters and the classical ground-based standard photometric systems, this fact enhances the importance of testing our ability to determine some physical properties of the observed stars directly in the natural XMM-OM colour system. In the present paper, we will show that it is possible to estimate both the temperature and the amount of interstellar absorption (or "reddening") for the hot stars that will be observed with XMM-OM.

The natural XMM-OM colour system can also be used for other investigations. For example, we have explored the possibilities to discriminate quasars from stars in multidimensional colour spaces based on the XMM-OM 

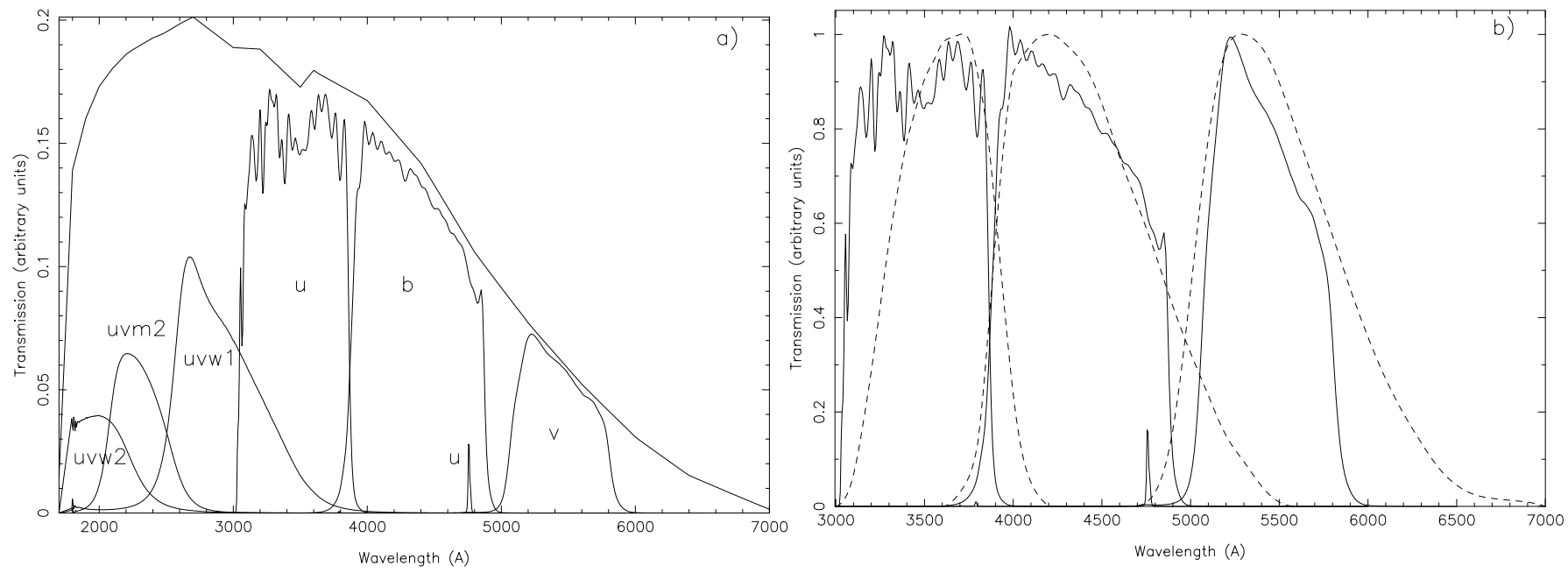

Fig. 1. a) XMM-OM's photometric system. The upper curve is the instrumental response in the absence of filter. It results from the product of the response curves of the detector, of the three mirrors and of the detector window. b) Comparison of the XMM-OM optical and the Johnson $U, B$ and $V$ filters. The pairs of filters are, from left to right: $u$ and $U ; b$ and $B ; v$ and $V$. The dashed curves are the Johnson filters

Table 1. Main characteristics of the XMM-OM filters. The wavelengths are expressed in $\AA$. $\lambda_{0}$ is the effective wavelength and $\lambda_{\max }$ is the position of the maximal transmission

\begin{tabular}{|c|c|c|c|}
\hline filter & $\lambda_{0}$ & $\lambda_{\max }$ & $F W H M$ \\
\hline uvw2 & 2070 & 2000 & $\sim 500$ \\
\hline uvm2 & 2298 & 2210 & 439 \\
\hline uvw1 & 2905 & 2680 & 620 \\
\hline$u$ & 3472 & 3270 & 810 \\
\hline$b$ & 4334 & 3980 & 976 \\
\hline$v$ & 5407 & 5230 & 684 \\
\hline
\end{tabular}

photometry. Nevertheless, the goal of this paper is not so much to provide the reader with exact analytical relations for these matters as to give qualitative results that will tell him how to observe and treat the data in order to get the best outcome, and what kind of results are to be expected.

In Sect. 2, we present the tools we used for the synthetic photometry. In Sect. 3, we discuss the colour transformations between the XMM-OM optical filters and the Johnson $U, B, V$ system. We treat the temperature and the interstellar absorption determination in Sects. 4 and 5 whereas the selection of quasar candidates is addressed in Sect. 6. Section 7 outlines the main conclusions of our study.

\section{The basic information and hypotheses}

\subsection{The filters}

All simulations presented hereafter were established on the basis of synthetic photometry. The filter passbands that we used for the XMM-OM photometric system are extracted from the XMM Users' Handbook (Dahlem \& Schartel 1999). Their transmission curves result from the product of the transmission curves and/or sensitivity curves of all physical devices in the XMM-OM light path. Namely, we have the filters themselves, three aluminized mirrors, one window at the entry of the detector and the detector response curve. All these curves originate from the same reference as above. The resulting passbands are plotted in Fig. 1a and their physical characteristics are summarized in Table 1. Throughout the rest of this paper, small characters refer to the XMM-OM filters and capital letters refer to the standard ones (i.e. the $U, B$ and $V$ taken from Bessel 1990).

The XMM-OM filter set consists of $3 \mathrm{UV}$ and 3 optical filters, further denoted by uvw2, uvm 2 , uvw1 and $u, b, v$ respectively, in order of increasing central wavelength. The $u, b$ and $v$ filters are intended to match the Johnson $U$, $B$ and $V$ (Bessel 1990) filters respectively, but they possess more or less rectangularly shaped passbands, which greatly affects the quality of the match and of the subsequent colour transformations between the two systems. This is especially true for what concerns the (mis)match between the Johnson $U$ and XMM-OM $u$ filters and thus the $(U-u)$ and $(U-B)$ related colour transformations. The XMM-OM $u$ band is characterized by:

- a strong sensitivity in the $3100-3300 \AA$ region, where the ground-based Johnson's $U$ is essentially blind;

- a reduced sensitivity in the critical Balmer decrement region $(3850-4100 \AA)$, leading to untransformability between both bands for stars showing Balmer jump (see Sects. 3.1.1, 3.1.6, 3.1.7). As we show later, $(U-u)$ can be evaluated thanks to uvw1.

Figure 1b allows comparison between XMM-OM and standard $U, B$ and $V$ filters. 
The UV XMM-OM filters do not correspond to any pre-existing standard system. No transmission measurements below $1800 \AA$ are given in the XMM flight simulator software files, so we added a point with $\sim 0 \%$ transmission at $1700 \AA$ in the UV filters.

\subsection{The stellar spectra}

The spectra we used for the synthetic photometry are those of the Kurucz' ATLAS 9 atmosphere models (Kurucz 1992), reddened by the Cardelli interstellar extinction model (Cardelli et al. 1989) with an average reddening law $\left(R_{V}=A_{V} / E(B-V)=3.1\right)$ when necessary. Except when otherwise stated, the set of spectra we chose mimics an unreddened solar composition main sequence (MS) since these 55 spectra possess the following characteristics: $A_{V}=0$.; $[\mathrm{Fe} / \mathrm{H}]=0$; $\log g=4$.; $3500 \mathrm{~K} \leq T_{\text {eff }} \leq 35000 \mathrm{~K}$. Stars we refer to as giants have $\log g=3.5$ (Allen 1976), those we refer to as metal poor stars have $[\mathrm{Fe} / \mathrm{H}]=-1.5$, typical for the halo of our Galaxy. The temperature interval between two adjacent Kurucz models varies along the tracks: it is $250 \mathrm{~K}$ below $10000 \mathrm{~K}, 500 \mathrm{~K}$ between $10000 \mathrm{~K}$ and $13000 \mathrm{~K}, 1000 \mathrm{~K}$ between $13000 \mathrm{~K}$ and $35000 \mathrm{~K}$, and $2500 \mathrm{~K}$ between $35000 \mathrm{~K}$ and $50000 \mathrm{~K}$.

Throughout the rest of this paper, the MS spectrum with $T_{\text {eff }}=9500 \mathrm{~K}$ was attributed a magnitude of zero in all filters.

All colour transformations established on the basis of the Kurucz spectra were compared with their equivalent obtained with the stellar spectral atlas prepared by Fioc \& Rocca-Volmerange (1997, hereafter FRV). This atlas comprises 65 spectra of stars of various luminosity classes (3 supergiants, 30 giants and 32 dwarfs) and spectral types $\left(T_{\text {eff }} \in[2500,180000] \mathrm{K}\right)$. It was constructed from observed spectra whenever possible (Gunn \& Strycker 1983; Heck et al. 1984), from synthetic spectra otherwise (Kurucz 1992; Clegg \& Middlemass 1987).

\subsection{The stellar locus for the quasar fields}

In Sect. 6, we analyse the ability of the XMM-OM photometric system to discriminate between quasars and stars. Therefore, we have to delimit both the quasar (see Sect. 2.4) and the stellar loci in the multidimensional colour space defined by the XMM-OM system. The stellar locus is defined by all the stars that are potential contaminants of the quasar candidate population. When looking at high galactic latitudes, the field stars are halo main sequence stars. We modelize their colours by integrating the emergent fluxes from Kurucz model atmospheres. We represent halo main sequence stars by the whole range of $T_{\text {eff }}, \log g=4$ and $[\mathrm{Fe} / \mathrm{H}]=-1.5$. Due to evolution, only the part for which $T_{\text {eff }} \leq 7000 \mathrm{~K}$ (corresponding to an early F spectral type) is still populated. These latter stars are the major constituents of the field stellar population. The reason why we consider the hotter stars as well is that their colours are close to those of other families of stars. We will use that property in Sect. 6, and discuss it further there.

Disk main sequence stars are also a possible contaminant in a list of quasar candidates. However, only the cool end $\left(T_{\text {eff }} \leq 7000 \mathrm{~K}\right)$ could be a problem and is taken into account. We chose to represent them by a $\log g=4$ and a solar metallicity. Indeed, the disk is rather thin: it has a typical scale height of $1 \mathrm{kpc}$, which corresponds to a distance modulus of 10 magnitudes. This means that disk OBA stars are too bright in apparent magnitudes to be mistaken for quasars. Another possible contaminant is constituted by halo giants. We represent them by Kurucz models with the following parameters: $T_{\text {eff }} \leq 7000 \mathrm{~K}, \log g=3$ and $[\mathrm{Fe} / \mathrm{H}]=-1.5$.

Some hotter stars are also present at high galactic latitudes as trace constituents but since they are bluer, a property they share with low-redshift quasars, they could constitute a strong contaminant in a quasar candidate list. We consider three families. The first one is the HorizontalBranch (HB) BA stars. These stars have $T_{\text {eff }} \geq 10000 \mathrm{~K}$ (up to $\sim 30000 \mathrm{~K}$ ). On the $\mathrm{HB}$, the gravity is strongly dependent on the effective temperature: we chose the dependency law we derived by averaging the ones quoted by Moehler et al. (1999) and by Conlon et al. (1991). According to Moehler et al. (1999), the best fit of Kurucz models to HB star's spectra is obtained for metal rich chemical compositions. We adopted $[\mathrm{Fe} / \mathrm{H}]=0.5$. As noticed by Miller \& Mitchell (1988), the population of HB stars corresponds to intrinsically bright objects and is observed to tail-off at faint magnitudes due to the finite dimension of the halo.

A second family is constituted of the subdwarfs of spectral type $\mathrm{OB}$ (sd OB). Their evolutionary status is still somewhat uncertain but they are usually associated to the Extended Horizontal Branch and to the evolution thereof (see Caloi 1989), although some authors refer to some of them as being post-AGB objects. We represent them by Kurucz models with $T_{\text {eff }}$ between $20000 \mathrm{~K}$ and $50000 \mathrm{~K}$ (see Conlon et al. 1991 but also Table 1 of Lenz et al. 1998), $\log g=5$ and solar metallicity.

Finally, the third family is made of degenerate stars, the so-called white dwarfs. Some models of degenerate stars exist but only a few have their emergent flux published. As a first approximation, degenerate stars are known to have $U B V$ colours very similar to black bodies. We therefore computed the colours of black bodies. In any case, this characteristic is perhaps not general and does not apply to the UV part of the spectrum. We finally used the emergent fluxes of the pure-hydrogen atmosphere models for degenerate stars of Koester (1999). We restricted ourselves to effective temperatures ranging from $7000 \mathrm{~K}$ to $80000 \mathrm{~K}$ and to $\log g=8.5$. We also integrated 
the spectra of the four white-dwarf primary spectrophotometric standards described by Bohlin et al. (1995) and the models of Wesemael et al. (1980). Both works are in good agreement (although not necessarily independent) with Koester's models.

\subsection{The quasar spectrum}

The quasar spectra used in Sect. 6 were derived from an average spectrum build from the composite spectra of Zheng et al. (1997) and Francis et al. (1991). The former was used from 310 to $2000 \AA$ and the latter from 2000 to $6000 \AA$. The match between both spectra is reasonably good, as shown by Zheng et al. (1997, their Fig. 9). It was performed on the continuum windows identified by these authors between 1400 and $2200 \AA$. The absorption characteristics of the quasar spectra due to the intervening Ly $\alpha$ clouds were established, between Ly $\alpha$ and the Lyman break, on the basis of the concept of Oke \& Korycansky (1982) and of the works presented by Irwin et al. (1991), Zuo \& Lu (1993) and Warren et al. (1994). For the region below the Lyman break, three absorption models were computed. The first two were adapted from the works of Møller \& Jakobsen (1990), Møller \& Warren (1991), Warren et al. (1994) and Giallongo \& Trevese (1990). The first one only takes into account the hydrogen clouds with column densities inferior or equal to $10^{17} \mathrm{~cm}^{-2}$ (sometimes called the Ly $\alpha$ forest clouds). It will further be referred to as model A. The second one additionally includes systems with column densities between $10^{17}$ and $10^{20} \mathrm{~cm}^{-2}$ (sometimes called the Lyman limit systems). It will further be referred to as model B. As an extreme case, we also considered a model where a strong (10 magnitudes) absorption occurs in the Lyman continuum at a redshift very close to the one of the quasar, absorption which persists in the whole observable Lyman continuum. This model will further be referred to as model C. These three models are strongly inspired by those described by Royer (1994) where full details can be found. To fix ideas, it is worth noticing that the higher normalization of Madau (1995) puts his model between our models B and C.

It must be clear that the quasar spectrum defined above is only representative of an average quasar and that some individual quasar spectra deviate strongly from it. The true population of quasars will exhibit some dispersion around the characteristics of this quasar. It is well known (see e.g. Francis et al. 1991) that the population of quasars displays a variety of power-law flux distributions and that the emission-line equivalent widths vary from one object to the other both in a systematic way (the Baldwin effect) and in a random way. On the basis of the power-law index dispersion reported, e.g. by Francis et al. (1991), we expect this effect to spread the colours of quasars by $\pm 0.3 \mathrm{mag}$ around our tracks. In addition, particular realizations of the distribution of high column density clouds along some line of sight could induce strong deviations from the mean behaviour.

\section{Colour transformations}

Colour transformations between a physical and a standard filter set always are a source of photometric errors (see e.g. Sterken \& Manfroid 1992 or Royer \& Manfroid 1996 and references therein). In the particular case of the XMMOM optical filters, the important discrepancies between the standard and non-standard $U, B$ and $V$ filters renders the choice of adequate transformations even more critical. In order to avoid additional errors introduced by subsequent colour computations, we established colour transformations for both single filters and colour indices.

Table 2. Summary of the colour transformations established in this paper. Capital letters refer to Johnson $U, B$ and $V$ filters. A one, two or three digit code $i_{1}, \ldots, i_{n}$ characterizes the transformation: $n$ is the number of temperature domains that have to be considered; $i_{k}$ is the order of the transformation in the $k^{\text {th }}$ domain (0 means no transformation is possible), e.g. 102 indicates that one has to divide the temperature domain in 3 distinct parts in which respectively linear, no and second order transformations are possible. The numbering of the domains is going from the hottest to the coolest stars

\begin{tabular}{|c|c|c|c|c|c|c|}
\hline & $U-u$ & $B-b$ & $V-v$ & $U-B$ & $B-V$ & $U-V$ \\
\hline$u-b$ & 202 & 102 & & 102 & & \\
\hline$u v w 1-u$ & 222 & & & & & \\
\hline$b-v$ & & $211 / 3$ & $111 / 2$ & & 1 & \\
\hline$u v w 1-b$ & & & & 102 & & \\
\hline$u-v$ & & & & & & $101 / 1$ \\
\hline$u v w 1-v$ & & & & & & $102 / 1$ \\
\hline
\end{tabular}

We restricted ourselves to a limited set of physically meaningful transformations, i.e. between equivalent or neighbouring filters in both filter sets. We nevertheless introduced transformations to the $(U-V)$ index in order to get colour transformations for colour indices that avoid the $b$ filter, which is the one for which the XMM-OM detector could be the most easily affected by saturation (XMM Users' Handbook, Eds. Dahlem \& Shartel 1999). We also established various transformations in which the $u v w 1$ filter supplants the $u$ filter. Indeed, contrary to the $u$ and $U$ filters, the $u v w 1$ filter is defined on the short wavelength side of the Balmer jump. This property renders the transformations based on uvw1 far better than those based on the neighbouring $u$ filter, i.e. they are more linear and essentially possible on a wider temperature range. As expected (Sect. 2.1), the uvw1 band can be used to correct the $(U-u)$ index in the critical domain where the Balmer jump is important (see Sect. 3.1.2).

The colour transformations presented below being established on synthetic spectra, it is obvious that they 
should be refined through actual observations of suitable standard fields with the satellite and from the ground. The main interest in the transformations given here is that they inform us about the kind of relations that are possible, and their validity domain.

The main characteristics of the considered colour transformations are summarized in Table 2. Two of them are illustrated in Figs. 2 and 3. The analytical forms of the transformations are given below, sorted by categories. Though some formal error bars are smaller, we did not indicate uncertainties smaller than $0.001 \mathrm{mag}$ in the relations given below.

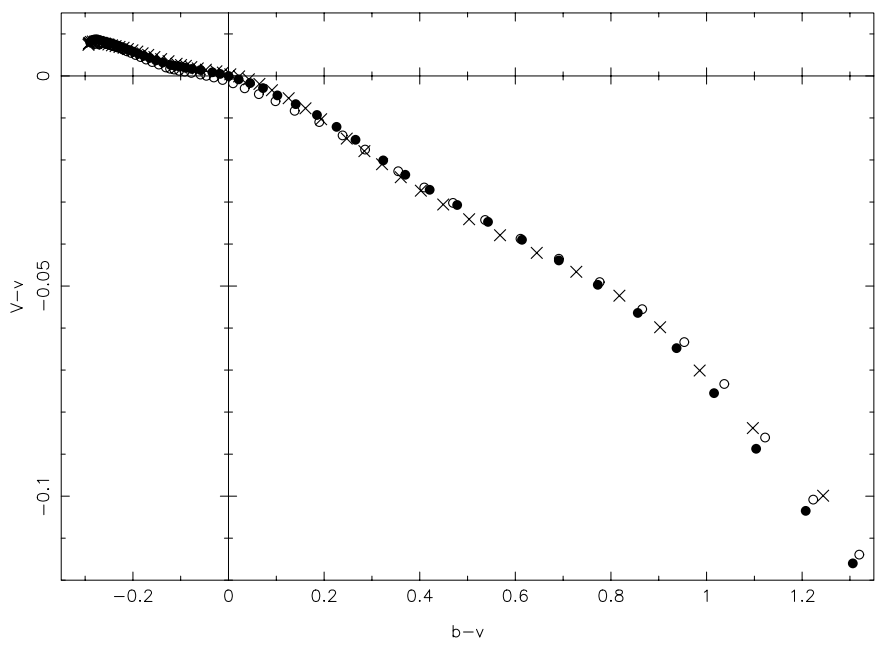

Fig. 2. The $(V-v)$ vs. $(b-v)$ diagram. Filled circles are MS stars, open ones are giants and crosses represent metal poor stars. The lowest temperature is $4000 \mathrm{~K}$ (bottom right) and the highest is $35000 \mathrm{~K}$ (upper left). The relation is monotonic and therefore the transformation is possible over the entire range

\subsection{Solar composition main sequence stars}

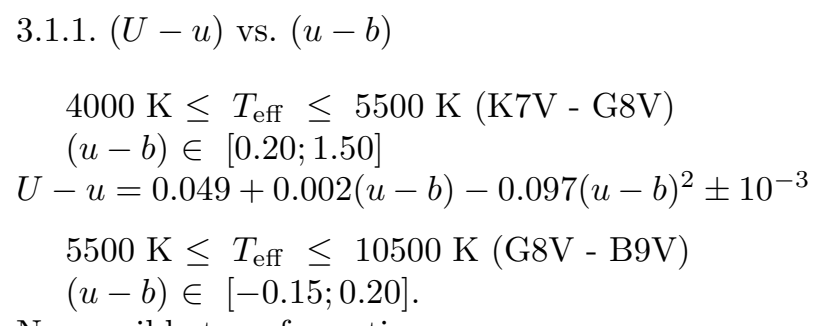

No possible transformation.

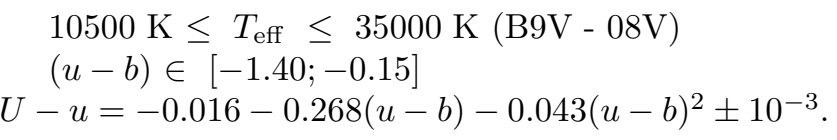

\subsection{2. $(U-u)$ vs. $(u v w 1-u)$}

$3500 \mathrm{~K} \leq T_{\text {eff }} \leq 7250 \mathrm{~K}(\mathrm{M} 3 \mathrm{~V}-\mathrm{F} 0 \mathrm{~V})$

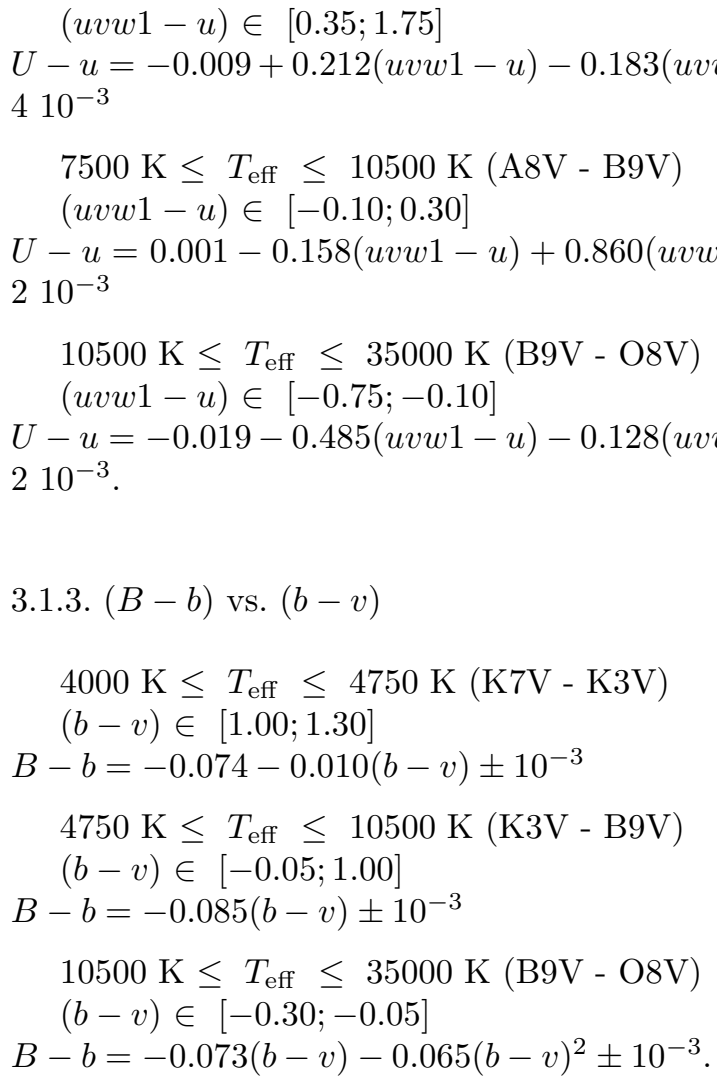

A third order fit can be applied to the whole interval:

$4750 \mathrm{~K} \leq T_{\text {eff }} \leq 35000 \mathrm{~K}(\mathrm{~K} 3 \mathrm{~V}-\mathrm{O} 8 \mathrm{~V})$

$(b-v) \in[-0.30 ; 1.00]$

$B-b=-0.070(b-v)-0.040(b-v)^{2}+0.026(b-v)^{3} \pm$ $10^{-3}$.

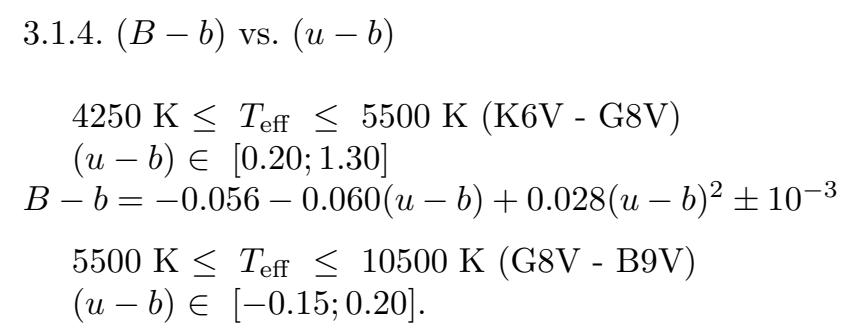

No possible transformation.

$10500 \mathrm{~K} \leq T_{\text {eff }} \leq 35000 \mathrm{~K}(\mathrm{~B} 9 \mathrm{~V}-\mathrm{O} 8 \mathrm{~V})$

$(u-b) \in[-1.40 ;-0.15]$

$B-b=0.003-0.009(u-b) \pm 10^{-3}$.

$$
\begin{aligned}
& \text { 3.1.5. }(V-v) \text { vs. }(b-v) \\
& 4000 \mathrm{~K} \leq T_{\text {eff }} \leq 5250 \mathrm{~K}(\mathrm{~K} 7 \mathrm{~V}-\mathrm{K} 0 \mathrm{~V}) \\
& (b-v) \in[0.85 ; 1.30] \\
& V-v=0.061-0.136(b-v) \pm 10^{-3} \\
& 5250 \mathrm{~K} \leq T_{\text {eff }} \leq 8750 \mathrm{~K}(\mathrm{~K} 0 \mathrm{~V}-\mathrm{A} 3 \mathrm{~V}) \\
& \quad(b-v) \in[0.07 ; 0.85] \\
& V-v=0.0025-0.068(b-v) \pm 10^{-3}
\end{aligned}
$$



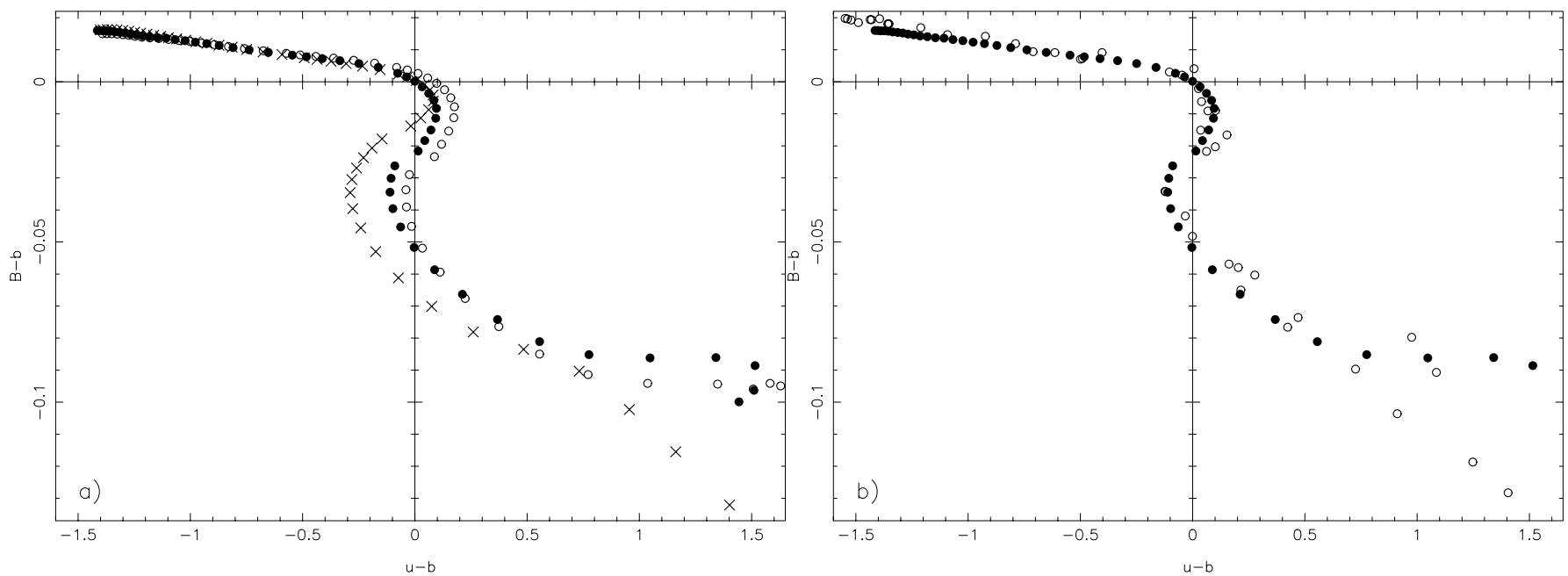

Fig. 3. a) $(B-b)$ vs. $(u-b)$ diagram. Symbols have the same meaning as in Fig. 2. Stars have effective temperatures between $3500 \mathrm{~K}$ and $35000 \mathrm{~K}$. This is a good illustration of a case where no transformation is possible in the middle part of the temperature domain. b) Same diagram for FRV MS and giant stars (open circles). Kurucz MS stars (filled circles) are given for comparison. Stars below $4000 \mathrm{~K}$ have been excluded from this plot

$$
\begin{gathered}
8750 \mathrm{~K} \leq T_{\text {eff }} \leq 30000 \mathrm{~K}(\mathrm{~A} 3 \mathrm{~V}-\mathrm{B} 0 \mathrm{~V}) \\
(b-v) \in[-0.30 ; 0.07] \\
V-v=-0.001-0.033(b-v) \pm 10^{-3} .
\end{gathered}
$$

A second order fit can be applied to the whole interval:

$$
4000 \mathrm{~K} \leq T_{\mathrm{eff}} \leq 30000 \mathrm{~K}(\mathrm{~K} 7 \mathrm{~V}-\mathrm{B} 0 \mathrm{~V})
$$$$
(b-v) \in[-0.30 ; 1.30]
$$

$V-v=-0.001-0.041(b-v)-0.035(b-v)^{2} \pm 210^{-3}$.

$$
\begin{aligned}
& \text { 3.1.6. }(U-B) \text { vs. }(u-b) \\
& \quad 4250 \mathrm{~K} \leq T_{\text {eff }} \leq 5500 \mathrm{~K}(\mathrm{~K} 6 \mathrm{~V}-\mathrm{G} 8 \mathrm{~V}) \\
& (u-b) \in[0.20 ; 1.35] \\
& \begin{array}{l}
\mathrm{U}-\mathrm{B}=0.120+1.087(u-b)-0.135(u-b)^{2} \pm 210^{-3} \\
5500 \mathrm{~K} \leq T_{\text {eff }} \leq 10500 \mathrm{~K}(\mathrm{G} 8 \mathrm{~V}-\mathrm{B} 9 \mathrm{~V}) \\
(u-b) \in[-0.15 ; 0.20] .
\end{array}
\end{aligned}
$$

No possible transformation.

$$
\begin{aligned}
& 10500 \mathrm{~K} \leq T-\mathrm{eff} \leq 35000 \mathrm{~K}(\mathrm{~B} 9 \mathrm{~V}-\mathrm{O} 8 \mathrm{~V}) \\
& (u-b) \in[-1.4 ;-0.15] \\
& U-B=0.003+0.819(u-b) \pm 510^{-3} .
\end{aligned}
$$

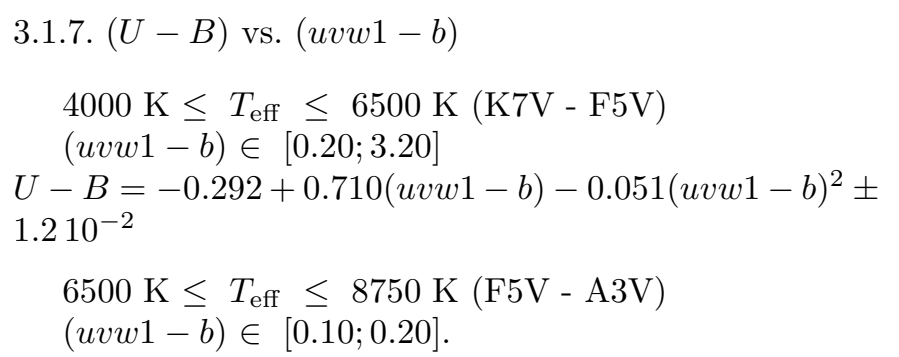

No possible transformation.

$$
\begin{aligned}
& 8750 \mathrm{~K} \leq T_{\text {eff }} \leq 35000 \mathrm{~K}(\mathrm{~A} 3 \mathrm{~V}-\mathrm{O} 8 \mathrm{~V}) \\
& (u v w 1-b) \in[-2.20 ; 0.10] \\
& U-B=0.002+0.528(u v w 1-b) \pm 810^{-3} .
\end{aligned}
$$

$$
\begin{aligned}
& \text { 3.1.8. }(B-V) \text { vs. }(b-v) \\
& 3500 \mathrm{~K} \leq T_{\text {eff }} \leq 35000 \mathrm{~K}(\mathrm{M} 3 \mathrm{~V}-\mathrm{O} 8 \mathrm{~V}) \\
& (b-v) \in[-0.30 ; 1.40] \\
& B-V=0.002+0.997(b-v) \pm 910^{-3} . \\
& \text { 3.1.9. }(U-V) \text { vs. }(u-v) \\
& 4000 \mathrm{~K} \leq T_{\text {eff }} \leq 6500 \mathrm{~K}(\mathrm{~K} 7 \mathrm{~V}-\mathrm{F} 5 \mathrm{~V}) \\
& (u-v) \in[0.40 ; 2.80] \\
& \begin{aligned}
U-V=0.133+0.947(u-v) \pm 2.310^{-2} \\
\\
6500 \mathrm{~K} \leq T_{\text {eff }} \leq 8500 \mathrm{~K}(\mathrm{~F} 5 \mathrm{~V}-\mathrm{A} 4 \mathrm{~V}) \\
(u-v) \in[0.20 ; 0.40] .
\end{aligned}
\end{aligned}
$$

No possible transformation, though the deviation from the general shape of the sequence is very slight (see below for further comments).

$$
\begin{gathered}
8500 \mathrm{~K} \leq T_{\text {eff }} \leq 35000 \mathrm{~K}(\mathrm{~A} 4 \mathrm{~V}-\mathrm{O} 8 \mathrm{~V}) \\
(u-v) \in[-1.70 ; 0.20] \\
U-V=0.008+0.843(u-v) \pm 810^{-3} .
\end{gathered}
$$

The general shape of our synthetic main sequence is pretty close to a straight line in the $(U-V)$ vs. $(u-v)$ diagram, so that, if precision is not critical (at most $0.1 \mathrm{mag}$ ), one can also use the following relation:

$$
\begin{aligned}
& 4000 \mathrm{~K} \leq T_{\text {eff }} \leq 35000 \mathrm{~K}(\mathrm{~K} 7 \mathrm{~V}-\mathrm{O} 8 \mathrm{~V}) \\
& (u-v) \in[-1.70 ; 2.80] \\
& U-V=0.100+0.930(u-v) \pm 5.710^{-2} \text {. } \\
& \text { 3.1.10. }(U-V) \text { vs. }(u v w 1-v) \\
& 4000 \mathrm{~K} \leq T_{\text {eff }} \leq 7000 \mathrm{~K}(\mathrm{~K} 7 \mathrm{~V}-\mathrm{F} 1 \mathrm{~V}) \\
& (u v w 1-v) \in[0.65 ; 4.50] \\
& U-V=-0.201+0.786(u v w 1-v)-0.029(u v w 1-v)^{2} \pm \\
& 1.910^{-2}
\end{aligned}
$$


$7000 \mathrm{~K} \leq T_{\text {eff }} \leq 7750 \mathrm{~K}(\mathrm{~F} 1 \mathrm{~V}-\mathrm{A} 7 \mathrm{~V})$

$(u v w 1-v) \in[0.50 ; 0.65]$.

No possible transformation. The problem is however very slight and seems to be due to the Kurucz synthetic spectra rather than to the filters. The best here is to use the general relation proposed below for the whole temperature range.

$7750 \mathrm{~K} \leq T_{\text {eff }} \leq 35000 \mathrm{~K}(\mathrm{~A} 7 \mathrm{~V}-\mathrm{O} 8 \mathrm{~V})$

$(u v w 1-v) \in[-2.45 ; 0.50]$

$U-V=0.004+0.577(u v w 1-v) \pm 910^{-3}$.

The same kind of considerations as for the $(U-V)$ vs. $(u-v)$ case holds concerning a general relation for the whole set of temperatures:

$$
\begin{gathered}
4000 \mathrm{~K} \leq T_{\text {eff }} \leq 35000 \mathrm{~K}(\mathrm{~K} 7 \mathrm{~V}-\mathrm{O} 8 \mathrm{~V}) \\
(u v w 1-v) \in[-2.45 ; 4.50] \\
U-V=0.023+0.596(u v w 1-v) \pm 410^{-2} .
\end{gathered}
$$

\subsection{Giants}

Whenever a colour transformation is possible for MS stars, it is generally also satisfactorily obeyed by giant stars. The difference essentially lies in the validity range of the colour transformations. Each time a forbidden zone appears in the temperature domain (i.e. each time there is a zero in Table 2), the transformation relative to the hottest stars remains valid for giant stars down to $\sim 250 \mathrm{~K}$ cooler than the lower temperature bound defined for MS stars. Symmetrically, the transformation relative to the coolest giant stars is only valid from stars $\sim 250 \mathrm{~K}$ cooler than the upper bound of the temperature interval defined for the corresponding MS star colour transformation (see Fig. 3a).

When colour transformations are possible for MS stars on the whole temperature domain, they are generally valid for giant stars as well (Fig. 2).

\subsection{Metal poor stars}

In this case, the situation is worse. Stars still obey the same relations as MS stars when colour transformations exist on the whole temperature domain. Even in other cases, the hottest stars still obey the same relations, but the range on which these transformations remain valid is now considerably diminished: the "hot" relations now hold only for $T_{\text {eff }} \geq 11500 \mathrm{~K}$ and the "cool" ones for $4750 \mathrm{~K} \geq T_{\text {eff }} \geq 4000 \mathrm{~K}$. Cool stars sometimes require different relations. This is true for the $(B-b)$ vs. $(u-b)$ as well as for both $(U-u)$ colour transformations (Fig. 3a).

\subsection{Comparison with observed spectra}

As a validity check, all colour transformations presented above were also derived on the basis of the mainlyobserved FRV spectra. When excluding stars cooler than
$4000 \mathrm{~K}$, the comparison between transformations established through Kurucz models and through FRV MS and giant star spectra is excellent, as illustrated in Fig. 3b. The latter ones are of course slightly more dispersed, but the dispersion is fully comparable to what could be expected on the basis of the transformations established in Sect. 3.2. Very small differences occur in some particular colour indices and are quite marginal: the $(B-b)$ colour index discriminates between the FRV MS and giant stars, but this only happens below $5000 \mathrm{~K} ;(V-v)$ is decreased by $\sim 0.01$ mag when calculated on the FRV spectra rather than on the Kurucz spectra; the Kurucz and FRV $(U-B)$ and $(U-V)$ colour indices are slightly discrepant below $5000 \mathrm{~K}$, as are the $(U-u)$ and the $(u v w 1-u)$ colour indices below $7000 \mathrm{~K}$.

As a conclusion, before the full inflight calibration on standard fields is performed and reduced, we recommend to use the relations given above to analyse the first data provided by the XMM-OM.

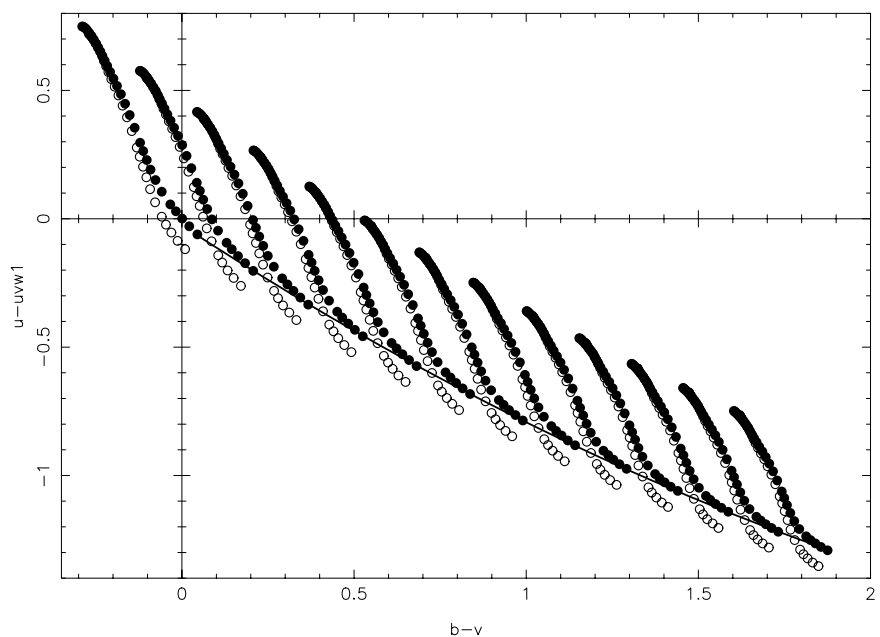

Fig. 4. The $(u-u v w 1)$ vs. $(b-v)$ colour diagram. Filled circles are MS stars, open ones are giants. Each "temperature track" (almost vertical) runs from $9000 \mathrm{~K}$ (bottom) to $35000 \mathrm{~K}$ (top). The reddening runs from $A_{V}=0$. (leftmost track) to $A_{V}=6$. (rightmost track) by steps of 0.5

\section{Reddening and temperature determination}

In order to discriminate between stars having different reddening and temperature characteristics, a combination of colour indices must be found such that temperature tracks (i.e. curves of constant reddening) do not cross each other in the related colour diagram. In the XMM-OM photometric system, we found only one fully suitable pair of such colour indices: $(u-u v w 1)$ and $(b-v)$. The corresponding colour diagram is shown in Fig. 4. This figure is representative of the weakest reddenings, but the diagram keeps its properties up to $A_{V} \sim 13$. Only a slight adaptation of the lowest temperature bound $(9000 \mathrm{~K} \rightarrow 11000 \mathrm{~K})$ is 

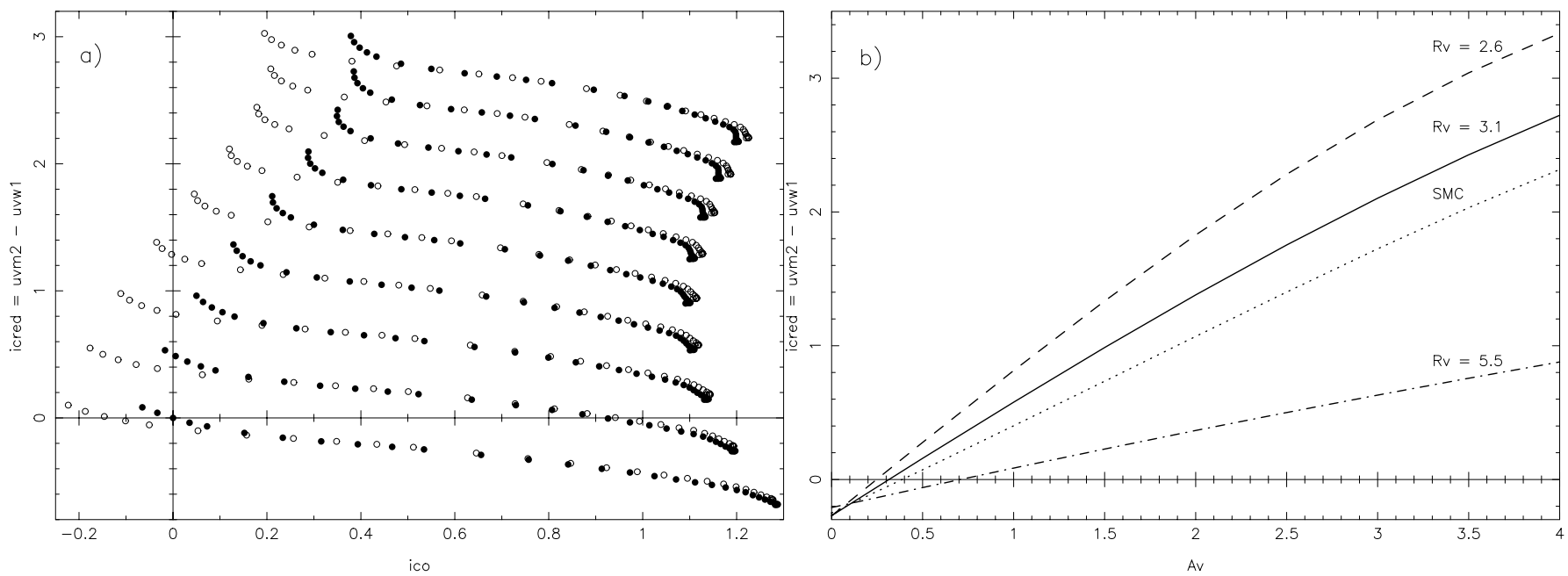

Fig. 5. a) The $i c_{\text {red }}$ vs. $i c_{0}$ colour diagram. Filled circles are MS stars, open ones are giants. Each temperature track runs from $9000 \mathrm{~K}$ (left) to $35000 \mathrm{~K}$ (right). The reddening runs from $A_{V}=0$. (bottom track) to $A_{V}=4.0$ (upper track). b) Dependency of the $A_{V}=A_{V}\left(i c_{0}, i c_{\text {red }}\right)$ function on the reddening law. This diagram shows a section at $i c_{0}=0.6$ in Fig. $5 \mathrm{a}\left(R_{V}=3.1\right.$, full line) and in each of the equivalent diagrams established for $R_{V}=2.6$ (dashed line), $R_{V}=5.5$ (dash-dotted line) and for the SMC reddening law (dotted line)

necessary. We did not plot stars below $9000 \mathrm{~K}$ in Fig. 4 because the temperature tracks of MS stars begin to overlay at this temperature, so that nothing can be said for lower temperatures. Uncertainties on the photometry will anyhow probably hamper any temperature determination below $10000 \mathrm{~K}$ (a $0.05 \mathrm{mag}$ photometric error can bring any $10000 \mathrm{~K}$ star on the $9000 \mathrm{~K}$ star locus). Above that value, the precision on the temperature determination depends on the temperature itself, but is typically of a few thousand Kelvins for colour indices accurate to 0.05 mag and for stars in the middle of the temperature domain. Such accuracy allows a reddening determination with a precision of about $\sim 0.3$ magnitudes in $A_{V}$ at any but the "coolest" temperatures $(<11000 \mathrm{~K})$.

The situation is slightly worse when one includes giant stars as well since the temperature tracks for the giants at a given reddening cross those of the less reddened MS stars. Even worse, they pass below the locus of the $9000 \mathrm{~K}$ MS stars at $\sim 10500 \mathrm{~K}$. As any place below this line can be occupied by stars with various combinations of temperature and reddening, the domain in which a temperature determination is possible for giants is restricted to stars above $\sim 11000 \mathrm{~K}$. As one does not know a priori whether or not a star is a giant, this of course also sheds some uncertainty on the temperature and reddening determination for MS stars below $\sim 12-13000 \mathrm{~K}$, where temperature tracks for MS stars and giants begin to significantly differ from each other.

Other discriminant colour indices than those of Fig. 4 exist in the system, but their combination with other ones only allows to determine the temperature and the reddening of the observed stars on much more restricted ranges of reddening and/or temperature. One can nevertheless design another, independent, reddening determination technique. In Fig. 4, reddening and temperature influence both colour indices plotted on the axes. As we will see now, one can nearly decouple these parameters and obtain better precision on the reddening. To do so, we need to define a colour index that is independent of reddening and another that is proportional to it. The former, that we will call $i c_{0}$, is defined as

$i c_{0}=(u v w 2-u v w 1)-\frac{E(u v w 2-u v w 1)}{E(u-u v w 1)}(u-u v w 1)$

where $E(x-y)$ stands for the colour excess of the $(x-y)$ colour index. $i c_{0}$ will remain a reddening free colour index as long as the colour excess ratio remains constant. This property is verified for stars with $T_{\text {eff }} \geq 9000 \mathrm{~K}$ and $A_{V} \leq 5.5$. Nevertheless, since $A_{2000} \sim 3 A_{V}$, four magnitudes of absorption in $V$ correspond to more than ten magnitudes of absorption in the uvm 2 and uvw2 filters. Hence, from now on, we will only consider the $A_{V} \leq 4$ domain. The mean $E(u v w 2-u v w 1) / E(u-u v w 1)$ colour excess ratio over this range is $-2.83( \pm 0.27)$. It is worth to note that $(u v w 2-u v w 1)$ and $(u-u v w 1)$ are, with $(u-b)$ and $(b-v)$, the only pairs of colour indices allowing the definition of a reddening free index over a reasonable range of stellar parameters.

In order to define the reddening dependent index, that we will call $i c_{\text {red }}$, the most obvious choice is the empirical $i c_{\text {red }}=u v m 2-(u v w 1+u v w 2) / 2$.

This index could be used but it is not really independent from $i c_{0}$ and its reddening dependence can be ameliorated. Indeed, though the uvm 2 filter stands on the $2175 \AA$ absorption bump, the uvw1 and uvw2 filters are not symmetric with respect to it, and of more importance, the uvw2 filter is significantly affected by the $2175 \AA$ absorption bump too. To refine the choice, we explored a large 

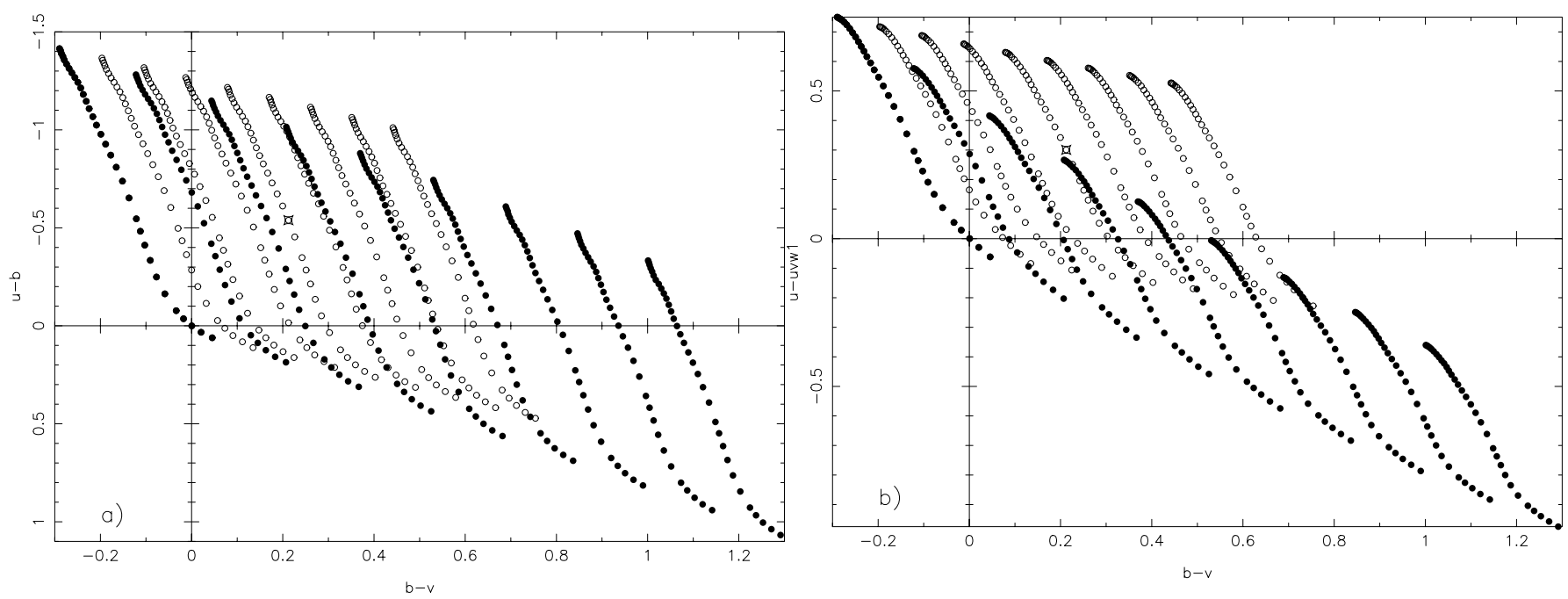

Fig. 6. a) The $(u-b)$ vs. $(b-v)$ colour diagram for MS stars reddened according to Cardelli et al. (1989) reddening law with $R_{V}=3.1$ (filled circles) and $R_{V}=5.5$ (open circles). Each temperature track runs from $9000 \mathrm{~K}$ (bottom) to $35000 \mathrm{~K}$ (top). The reddening runs from $A_{V}=0$. (leftmost track) to $A_{V}=4.0$ (rightmost track). The additional diamond symbol points to the star with $T_{\text {eff }}=15000 \mathrm{~K}, A_{V}=2$. and $R_{V}=5.5$ (see text). b) The $(u-u v w 1)$ vs. $(b-v)$ colour diagram with the same conventions

number of UV magnitude combinations and were finally brought to the conclusion that the best choice in terms of simplicity and dynamics of the index (and hence in terms of accuracy) is

$i c_{\text {red }}=u v m 2-u v w 1$.

There is in fact a wide variety of reddening-dependent indices in the XMM-OM system and, to give just another very simple one, (uvw2-uvw1) is nearly as good as (uvm $2-u v w 1)$. Even $(b-v)$ could be used as reddeningdependent index, so that the whole treatment that we carry on here on the UV filters could be performed on the $u b v$ filters as well. Nevertheless, it is important to note that, although the reddening-free index based on the optical filters is much better than the one based on the ultraviolet filters $(E(u-b) / E(b-v)=0.80 \pm 0.02$; compare the error bar with the UV case), the dynamic of the $(b-v)$ index is smaller than the one of the (uvm2-uvw1) index. Consequently, at equivalent accuracy on the photometry, the reddening determined thanks to the UV filters will be more accurate than the one obtained on the basis of the optical photometry (except for temperatures lower than $10000 \mathrm{~K}$ or higher than $25000 \mathrm{~K}$ ). If UV and optical photometries are available, reddening determinations in both domains should of course be used as a quality check. Indeed, as we will see in Sect. 5, the simultaneous use of visible and UV data allows a control of the consistency of the adopted reddening law.

Although lots of reddening dependent indices exist, none is perfectly independent from temperature and from $i c_{0}$ so that no simple $A_{V}=A_{V}\left(i c_{\text {red }}\right)$ relation can be drawn. Instead, one has to perform a bi-dimensional fit over the $\left(i c_{0}, i c_{\text {red }}\right)$ plane (Fig. 5a) to get the $A_{V}=$ $A_{V}\left(i c_{0}, i c_{\text {red }}\right)$ relation. We do not present this fit here since its detailed analytical form can only be usefully ob- tained through actual in-orbit satellite calibrations. The reddening dependency of $i c_{\text {red }}$ is illustrated in Figs. 5a and $5 \mathrm{~b}$. The solid line in Fig. 5b represents a section of the colour diagram shown in Fig. $5 \mathrm{a}$ at $i c_{0}=0.6$.

This reddening determination method, based on a reddening free and a reddening dependent index, is more accurate than what can be expected with the procedure outlined in Fig. 4 since error bars of 0.2 and $0.05 \mathrm{mag}$ on $i c_{0}$ and $i c_{\text {red }}$ respectively lead to $\leq 0.15$ mag uncertainty on $A_{V}$. On the other hand, one can see by comparing Fig. 5a and Fig. 4 that the uncertainties on $A_{V}$, due to the fact that the temperature tracks for MS and giant stars diverge at the coolest temperatures in the $(u-u v w 1)$ vs. $(b-v)$ colour diagram, are partly removed here for the weakest reddenings. Nevertheless, the colour diagram shown in Fig. 4 remains necessary since the $A_{V}=A_{V}\left(i c_{0}, i c_{\text {red }}\right)$ fit is not valid for all temperatures, so that we need an independent determination of temperature, precisely allowed by Fig. 4 .

\section{Non-standard reddening laws}

The reddening determination method presented in the previous section does not take into account the possibility of a non-standard reddening law. We will now address the following question: can we determine whether or not the reddening of a star observed with the XMM-OM is anomalous? To answer this, we integrated the same spectra with alternative values of $R_{V}$. Instead of $R_{V}=3.1$, - the standard value for diffuse interstellar medium and average value for the LMC-, we used $R_{V}=2.6$ and $R_{V}=5.5$ (observed by Cardelli et al. 1989, in the direction of HD 204827 and HD 37022 respectively). We also considered the peculiar UV absorption law established by 
Prévot et al. (1984) for the SMC. This reddening law does not show any absorption bump around $2175 \AA$. It is specific to the ultraviolet and was thus only applied to the UV filters.

Figures $6 \mathrm{a}$ and $6 \mathrm{~b}$ show the resulting $(u-b)$ vs. $(b-v)$ and $(u-u v w 1)$ vs. $(b-v)$ colour diagrams in the $R_{V}=5.5$ case. Let us consider a $15000 \mathrm{~K}$ star affected by 2 magnitudes of absorption (in $V$ ) with a reddening law characterized by such a high value of $R_{V}$ and let us see what will happen if we try to interpret the observed colours assuming a classical reddening law with $R_{V}=3.1$. In the $(u-b)$ vs. $(b-v)$ diagram, the colours mimic those of a slightly hotter star, affected by hardly more than one magnitude of absorption but in $(u-u v w 1)$ vs. $(b-v)$, the star falls out of the "authorized" range of colours. This discrepancy will clearly reveal that the assumption was wrong and that the star suffers from a peculiar absorption law. Figure 6 thus reveals that $(u-u v w 1),(b-v)$ and $(u-b)$ can be used to determine $T_{\text {eff }}, A_{V}$ and $R_{V}$ just as Fig. 4 shows that $(u-u v w 1)$ vs. $(b-v)$ can be used to determine $T_{\text {eff }}$ and $A_{V}$ once $R_{V}$ is known.

Of course, peculiar absorption laws also influence $i c_{0}$ and $i c_{\text {red }}$. As expected from Fig. 6, the colour excess ratios are reddening law dependent. Considering $0 \leq A_{V} \leq 4$., we found the mean value of $E(u v w 2-u v w 1) / E(u-u v w 1)$ to vary between -2.83 and -3.68 for $R_{V} \in[2.6 ; 5.5]$ (the value for the Prévot et al. 1984 reddening law is -3.04). Consequently, the appropriate $\left(i c_{0}, i c_{\text {red }}\right)$ has to be used once $R_{V}$ has been determined. Figure $5 \mathrm{~b}$ shows the influence of the reddening law variations on the $\left(i c_{0}, i c_{\text {red }}\right)$ diagram through comparison between $i c_{0}=0.6$ sections performed in the various realizations of that diagram.

As a conclusion, regarding the reddening determination, the main advantages of the XMM-OM UV filters are that

- at equivalent photometric accuracy, they allow a better determination of the amount of interstellar extinction than what is achievable with the optical filters only;

- they allow discrimination between standard and nonstandard extinction laws; this is of primary importance since interpretation of X-ray data requires knowledge of the column density along the line of sight, what is often estimated through reddening measurements (e.g. Bohlin et al. 1978).

\section{The XMM-OM multicolour space and quasars}

In the present section, we investigate the ability of the XMM-OM photometric system to segregate quasars from stars on the basis of their colours in the multicolour space definable from the set of the different filters used. Therefore, we integrated both the stellar spectra discussed in Sect. 2.3 and the average quasar spectra discussed in Sect. 2.4. The latter ones have been considered at redshifts from 0.0 to 4.4 by steps of 0.1 . We start our analysis with the XMM-OM version of the classical $(U-B)$ vs. $(B-V)$ colour diagram.

\subsection{The basic $(u-b)$ vs. $(b-v)$ diagram}

The $(U-B)$ vs. $(B-V)$ colour diagram is probably one of the most widely used in astronomy. Therefore, Fig. 7 presents the XMM-OM version $((u-b)$ vs. $(b-v))$ of this diagram. Fortunately, no huge difference appears between the two versions and the XMM-OM colour diagram retains most of the properties of its classical counterpart.

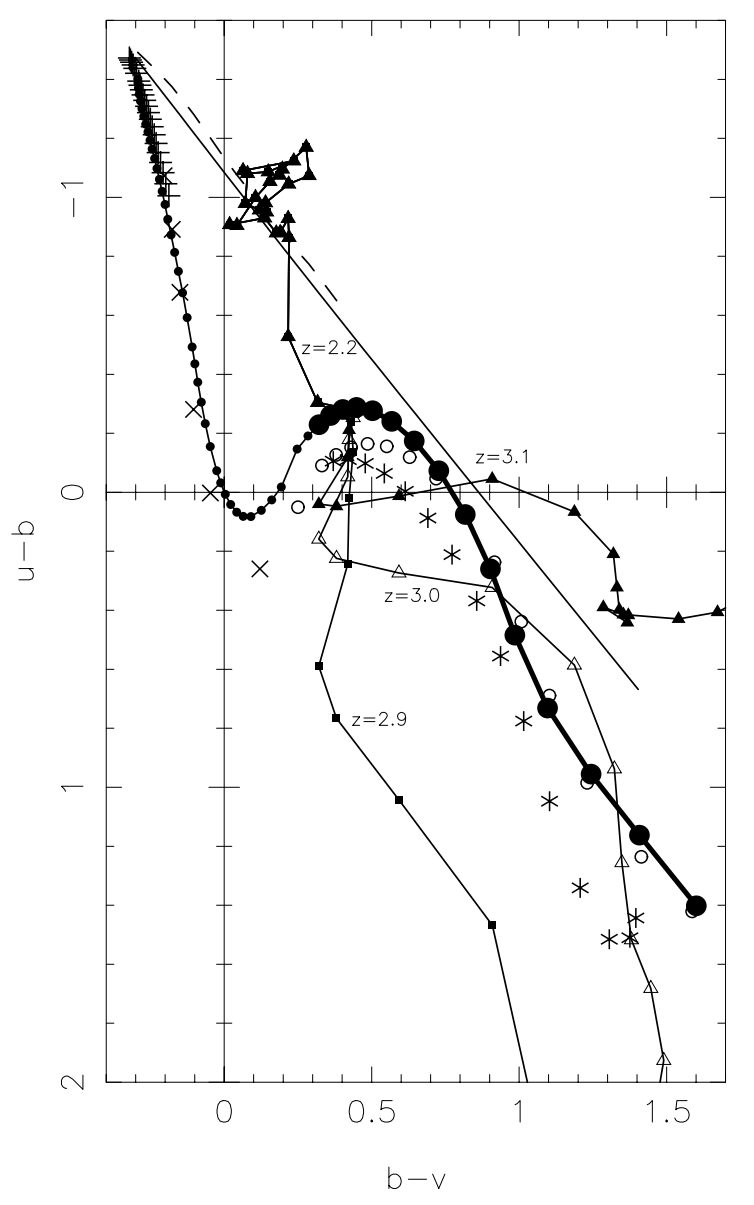

Fig. 7. The $(u-b)$ vs. $(b-v)$ colour diagram. The halo main sequence stars are represented by filled circles; the part of the sequence populated in our Galaxy is in bold character. The halo giants are represented by open circles whereas the cool end of the disk main sequence is described by asterisks. The crosses stand for the HB BA stars whereas the plus signs deal with the sd OB stars. The continuous line represents the black body locus and the dashed line the positions of the degenerate stars as derived from Koester's models. Model A average quasar spectrum has its redshift track punctuated with filled triangles every 0.1 in redshift whereas model B is marked out by open triangles and model $\mathrm{C}$ by filled squares. The highest plotted redshifts are 4.1, 3.7 and 3.1 for the quasars of models $\mathrm{A}, \mathrm{B}$ and $\mathrm{C}$ respectively 
The locus of the theoretical halo main sequence stars is given (filled circles). The classical potential-well shape of the curve outlining the effect of the Balmer continuum is clearly visible. The part below the turn-off represents the stars that are still on the main sequence in the halo of our Galaxy; it is represented by a bold line. This bold line is the locus of the majority of the field stars which are the objects against which we have to perform the basic discrimination when looking for quasars. The locus of cool giants is also given (open circles) and is very similar to the previously discussed one. On the other hand, the disk main sequence (spectral types FGKM), which could also provide some confusion, is visible below the halo main sequence (asterisks). The HB stars (crosses) have colours very similar to the hot part (astronomically non populated) of the halo main sequence. This is also true for the sd OB stars (plus signs). These objects are classical contaminants of the samples of quasar candidates selected on the basis of the $U / B$ excess. Another contaminant are the degenerate stars. The black body line is also given in Fig. 7 and the Koester's models whose spectrum we integrated fall close to this line. Figure 7 also exhibits the track of our average quasars as a function of redshift. For redshifts $z \leq$ 2.1 , the average quasars are wandering around $u-b=-1.0$ and $b-v=0.15$. This is 0.2 magnitude bluer in $u-b$ than in the case of the standard system (see e.g. Fig. 6 of Cristiani \& Vio 1990 and Fig. 9 of Moreau \& Reboul 1995). This ability to better detect the bluer objects is essentially due to the comparatively high transmission of the $u$ filter below $3200 \AA$ (see Fig. 1b and Sect. 2.1).

The wandering around the mean place is essentially due to the various emission lines entering and going out of the different filters but also partly to the particular shapes of the top of the transmission curves. The region around this mean place is also where one can find degenerate stars (with typical $T_{\text {eff }} \sim 15000 \mathrm{~K}$ ) and the presently investigated colour combination is not useful in discriminating between both kind of objects. At $z=2.2$, the quasars have left their low-redshift location to move almost parallel to the $(u-b)$ axis. This is due to the fact that the Ly $\alpha$ emission line is leaving the $u$ filter to enter the $b$ one and is progressively replaced by the Ly $\alpha$ forest. This occurs 0.07 earlier in redshift than in the Johnson standard system.

The tracks of the average quasars are also given for higher redshifts. It should however be clearly stated that for redshifts $z \geq 2.9$, both the $u$ and the $b$ filters are mainly sampling the Ly $\alpha$ forest and the related quasar location in the bidimensional (2D) colour diagram is highly model dependent. It is interesting to notice that the model B spectra tend to follow the stellar locus whereas model $\mathrm{C}$ quasars stay bluer in $b-v$. In any case, the discrimination is essentially possible for low-redshift $(z \leq 2.2)$ quasars and for high-redshift weakly or strongly absorbed objects.

Figure 8 gives the distance (in this two-dimensional space) between the model A quasar and the stellar locus as a function of redshift (by steps of 0.1 , filled circles). The stellar locus adopted here is the whole halo main sequence. Except for degenerate stars, this locus can be considered as representative of most of the other potential contaminants.

Figure 8 clearly demonstrates the ability to discriminate between non-degenerate stars and quasars with redshifts $z \leq 2.2$. In the range $z \in[2.2, \sim 3$.] (or more), quasars have $u b v$ colours very similar to stars and the discrimination power will only improve through the use of additional filters. The apparent improvement for redshifts in the range $z \in[3.3,3.6]$ is not present for model $\mathrm{B}$ quasars: this pinpoints the model dependent character of this particular result.

At $z>3.8$, all three filters are essentially in the Ly $\alpha$ forest and the increase in distance is indicative of a potential discrimination but the latter is bound to be highly dependent on the particular realization of the distribution of the Ly $\alpha$ absorbers both in redshift and in density. The completeness of the related sample will be hard to ascertain.

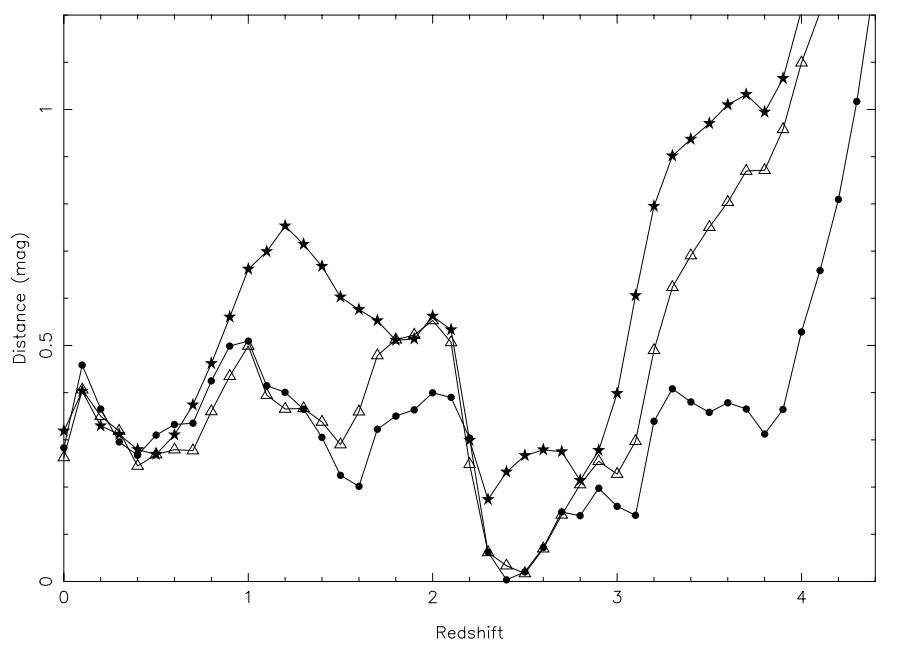

Fig. 8. The reduced distance between the average model A quasar and the stellar locus as a function of redshift, by steps of 0.1 . The first curve (circles) represents the two-dimensional distance in the $2 \mathrm{D}(u-b)$ vs. $(b-v)$ colour diagram as given in Fig. 7. The second curve (triangles) represents $\sqrt{2} / \sqrt{3}$ times the three-dimensional distance in the $3 \mathrm{D}(u v w 1-u)$ vs. $(u-b)$ vs. $(b-v)$ colour diagram. The third curve (stars) gives $\sqrt{2} / \sqrt{4}$ times the four-dimensional distance in the $4 \mathrm{D}(u v w 2-u v w 1)$ vs. $(u v w 1-u)$ vs. $(u-b)$ vs. $(b-v)$ colour diagram

\subsection{Adding uvw1}

Our simulations concerning the uvw1 filter clearly indicate that this filter is roughly as sensitive to the Balmer continuum (not to confuse with the Balmer jump) as the $u$ filter. The $(u v w 1-b)$ colour index of stars has a behaviour 
very similar to the $(u-b)$ one and the locus of stars in a $(u v w 1-b)$ vs. $(b-v)$ diagram is very reminiscent of Fig. 7. The $(u v w 1-u)$ colour index is much less sensitive to the Balmer continuum. It is interesting to notice that in a $2 \mathrm{D}(u v w 1-u)$ vs. $(u v w 1-v)$ colour diagram, the quasars with $z \leq 1.5$ are perfectly superimposed on the locus of stars. Therefore, this combination is not interesting for low-redshift quasars but quasars with redshifts between 1.6 and 2.1 are moving away from the stellar locus in the same diagram. This is essentially due to the Ly $\alpha$ emission line leaving $u v w 1$ for the $u$ filter and to the Ly $\alpha$ forest becoming dominant in uvw1. Nevertheless, full exploitation of this phenomenon requires observations in the $b$ filter. This is particularly striking in the $(u v w 1-u)$ vs. $(u-b)$ colour diagram (not shown here).

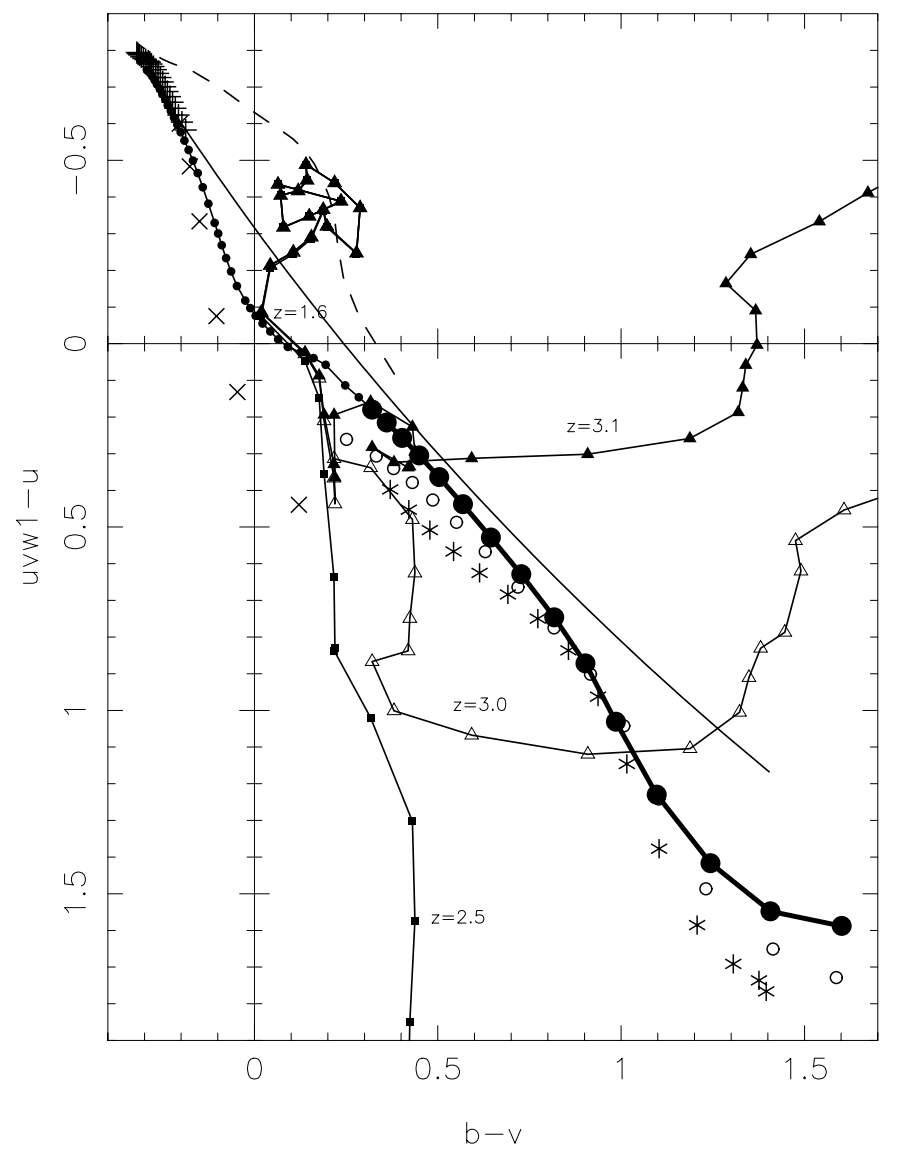

Fig. 9. The $(u v w 1-u)$ vs. $(b-v)$ colour diagram. The symbols have the same meaning as those used for Fig. 7. The highest plotted redshifts are 4.1, 3.9 and 2.6 for the quasars of models $\mathrm{A}, \mathrm{B}$ and $\mathrm{C}$ respectively

Figure 9 gives the $2 \mathrm{D}(u v w 1-u)$ vs. $(b-v)$ colour diagram. At redshifts $z \leq 1.5$, quasars are wandering around $u v w 1-u=-0.4$ and $b-v=0.15$. This is slightly aside the non-degenerate stellar locus and the uvw1 filter contributes, although weakly, to the star-quasar separation. For redshifts $1.6 \leq z \leq 3.0$, the average quasar joins the stellar locus in this 2D diagram of Fig. 9 but it is known to deviate from the stellar locus in the $(u v w 1-u)$ vs. $(u v w 1-v)$ colour diagram for $1.6 \leq z \leq 2.1$. In Fig. 8 is also given the distance between the quasar and the stellar locus in the three-dimensional space $(u v w 1-u)$ vs. $(u-b)$ vs. $(b-v)$. Increasing the number of dimensions of the space always brings an increase of the distance between objects, although the effect is purely geometrical. To test whether or not the added filter brings a strategical contribution due to its location in the wavelength domain, one has to compare the distances reduced to the lower dimension space. Therefore, in Fig. 8, we compare the two-dimensional true distance (in $(u-b)$ vs. $(b-v)$ ) to the reduced $3 \mathrm{D}$ distance which is the three-dimensional true distance $($ in $(u v w 1-u)$ vs. $(u-b)$ vs. $(b-v))$ multiplied by a $\sqrt{2} / \sqrt{3}$ factor.

From Fig. 8, it is absolutely clear that the main contribution of the use of uvw1 to the discriminating power of the XMM-OM photometry is essentially located at the redshift range 1.6 to 2.1. It is also interesting to notice that low-redshift quasars are wandering in Fig. 9 slightly aside the black body line. However, the degenerate stars do not follow the black body locus but, rather, are again mixed with low-redshift quasars (a typical effective temperature for a white dwarf in the middle of the low-redshift quasar locus is $12000 \mathrm{~K}$ ). This suggests that the discrimination between degenerate stars and quasars is bound to remain poor. Beyond $z=3.0$, the model A quasars seem to remain out of the stellar locus, and the model $\mathrm{C}$ quasars stay bluer in $b-v$. This again depends on the particular behaviour of the $\operatorname{Ly} \alpha$ absorbers in the line of sight of the observed quasar.

\subsection{Adding uvw2}

Filter uvw2 could also be used to build-up colour diagrams. However, it should be kept in mind that the $\mathrm{XMM}-\mathrm{OM}$ is not very sensitive in this passband and the precision of the measurement in uvw2 could be markedly worse than in any of the other filters. The use of filter $u v w 2$ is illustrated in Fig. 10 where the $2 \mathrm{D}(u v w 2-u v w 1)$ vs. $(b-v)$ colour diagram is given. Similarly to the previous case, low-redshift $(z \leq 0.6)$ quasars are wandering at $(u v w 2-u v w 1)=-0 . \overline{3}$ and, of course, $(b-v)=0.15$. This is slightly out of the stellar locus. However, at $z \sim 0.7$, the average quasars progressively become redder in $(u v w 2-u v w 1)$ due as usual to the $\operatorname{Ly} \alpha$ emission line going from the first filter to the second. The present colour index is expected to be discriminant when the $\operatorname{Ly} \alpha$ line is located in the $u v w 1$ filter, i.e. roughly for redshifts between 0.8 and 1.6. This is easily seen in the $(u v w 2-u v w 1)$ vs. $(u v w 1-u)$ colour diagram as well as in the (uvw2 - uvw1) vs. (uvw1-b) one; this pinpoints the importance of the joint use of the $u$ filter (or perhaps the $b$ one) along with the pair $u v w 2$, 


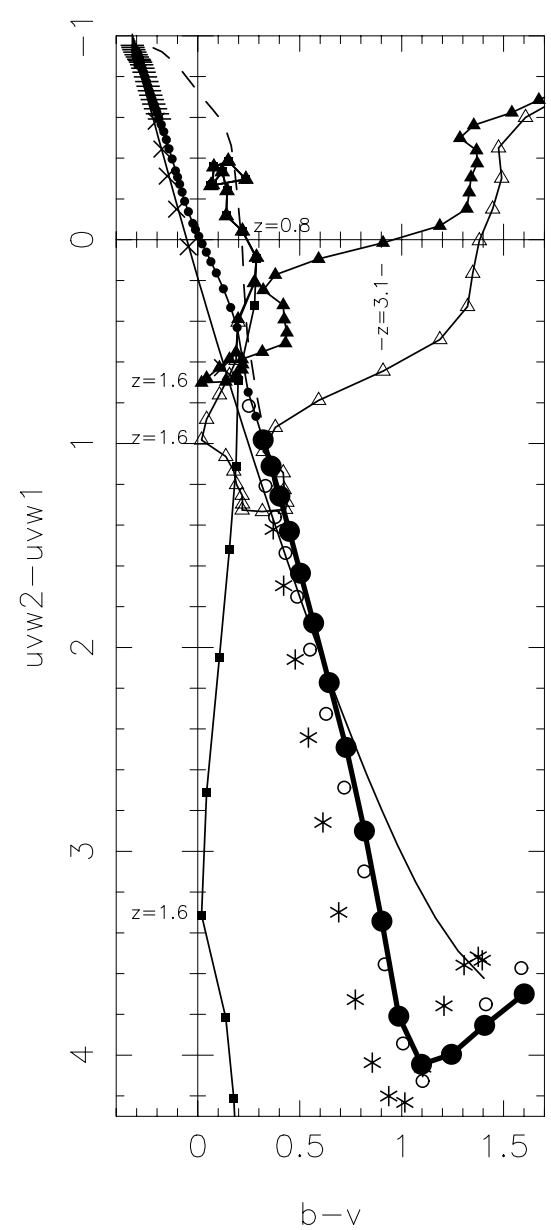

Fig. 10. The $(u v w 2-u v w 1)$ vs. $(b-v)$ colour diagram. The symbols have the same meaning as those used for Fig. 7. The highest plotted redshifts are 4.1, 3.9 and 1.8 for the quasars of models $\mathrm{A}, \mathrm{B}$ and $\mathrm{C}$ respectively

uvw1. Figure 8 exhibits the reduced $(\sqrt{2} / \sqrt{4}=1 / \sqrt{2})$ four-dimensional distance in the $4 \mathrm{D}(u v w 2-u v w 1)$ vs. $(u v w 1-u)$ vs. $(u-b)$ vs. $(b-v)$ colour space. It is clear that the contribution of the uvw2 filter contrasting with the $u v w 1$ one is increasing the discrimination power in the redshift range 0.8 to 1.6 . This effect could help in generating quasar samples that are more homogeneous in redshift since the use of the new filters alleviates the well-known bias of $U / B$ selected quasar candidates due to the presence of a strong $\mathrm{C}$ IV line in the $B$ filter (at $z \sim 1.6-1.7$ ). From Fig. 10, it is again clear that the degenerate stars do not follow the black body line and that they still remain a strong contaminant of the samples of quasars (particularly around $T_{\text {eff }} \sim 13000 \mathrm{~K}$ ).

\subsection{General considerations}

From Fig. 8, one can conclude that the XMM-OM filter set is good at discriminating between non-degenerate stars and quasars at low redshifts $(z \leq 2.2)$. This is particularly true in the range 0.8 to 2.1 where the use of the $u v w 1$ and $u v w 2$ filters allows a significantly better discrimination that is even able to wash out the decrease in efficiency around $z \sim 1.6-1.7$ sometimes exhibited by traditional $(U-B)$ vs. $(B-V)$ surveys. For very low redshifts $(z<0.8)$, the advantage of this photometric system is less marked. However, one should not forget that Fig. 8 gives the reduced distance. Indeed, the minimum true distance between the quasar and the stellar locus is, in the $4 \mathrm{D}$ space of Sect. 6.3, somewhat larger than 0.35 magnitudes (occuring at $z=0.5$ ); this already implies a real possibility of segregation. For redshifts between 2.3 and 3.5, the selection is essentially inefficient, as for ground-based surveys neglecting the use of the $R$ and $I$ filters (for example). It is beneficial to recall that XMM-OM was originally designed with a red optical path that has been abandoned in the meantime. Beyond $z=3.5$, the average quasar is usually off the stellar locus but this corresponds to the presence of the Ly $\alpha$ forest in most of the filters and is thus again highly dependent on the particular realization of the Ly $\alpha$ absorption (density and actual locations of the strong Ly $\alpha$ absorbers on the line of sight). In addition, the flux below Ly $\alpha$ is comparatively much lower implying a far less precise photometry. Generally, it is clear that the XMM-OM filter set is not adapted to the study of high-redshift quasars: although some of them will be easily spotted, the selection criterion will remain inhomogeneous. On the other hand, the XMM-OM photometry has no discrimination power between degenerate stars and quasars. Particularly for white dwarfs with effective temperatures in the range $12000 \mathrm{~K}-15000 \mathrm{~K}$, the colours are very similar and the domain in effective temperature is too small to authorize a proper segregation.

As a last point, we would like to recall the existence of the uvm 2 filter which has already been used to define the $i c_{\text {red }}$ index. We found no combination where this filter could be of some help to improve the situation. For example, in a $2 \mathrm{D}(u v w 2-u v m 2)$ vs. (uvm $2-u v w 1)$ colour diagram, the quasars are well located on the stellar locus except perhaps for redshifts around $z \sim 0.8 \rightarrow 1.0$ where they leave the stellar locus but this brings no strong improvement compared to the previously analysed filters.

\section{Conclusions}

In the present paper, we discussed the properties of the natural photometric system of the Optical Monitor onboard the X-ray Multi-Mirror satellite. On the basis of numerical simulations, we investigated its transformability to the standard Johnson $U B V$ system. We gave the main transformation equations both for individual filters and for colour indices. On the basis of the same technique of simulations, we showed that, for stars with effective temperatures higher than $9000 \mathrm{~K}$ observed with this system, it is possible to determine the temperature and the reddening (due to interstellar extinction) independently, as well 
as to detect non-standard reddening laws on given lines of sight. Finally, we made a detailed study of the possibilities to select quasar candidates on the basis of their location in the multicolour space definable from this particular photometric system. In particular, the interest of the use of the UV filters has been critically evaluated. The main conclusion of this analysis is that the use of the $u v w 1$ and $u v w 2$ filters allows to greatly enhance the non-degenerate stars quasars discrimination in the $[0.8 ; 2.1]$ redshift range. For lower redshifts, the discrimination remains reasonably efficient. The advantage to make use of the XMM-OM photometric system is nevertheless less clear for what concerns the higher redshifts $(z \geq 2.3)$ and the discrimination between the quasars and the degenerate stars. The quasar candidate selection could benefit from the addition of criterions based on the $\mathrm{X} /$ optical flux ratio, as these are usually larger for QSO/AGN than for stars (Stocke et al. 1991; Schmidt et al. 1998). The full treatment of the quasar candidate selection based on XMM observations is beyond the scope of the present paper, dedicated to the properties of the XMM-OM photometric system, and will be discussed elsewhere.

Acknowledgements. The authors are greatly indebted to D. Koester for the unpublished degenerate star models he kindly provided and to W. Zheng and P. Francis for a computer readable version of their composite quasar spectra. The authors also want to express their thanks to the Fonds National de la Recherche Scientifique (Belgium) for multiple supports. This research is supported in part by contract ARC 94/99-178 "Action de recherche concertée de la Communauté Française" (Belgium). Partial support through the PRODEX XMM-OM Project is also gratefully acknowledged.

\section{References}

Allen C.W., 1976, Astrophysical Quantities. Athlone Press London

Bessel M.S., 1990, PASP 102, 1181

Bohlin R.C., Savage B.D., Drake J.F., 1978, ApJ 224, 132

Bohlin R.C., Colina L., Finley D.S., 1995, AJ 110, 1316

Caloi V., 1989, A\&A 221, 27

Cardelli J.A., Clayton G.C., Mathis J.S., 1989, ApJ 345, 245

Clegg R.E.S., Middlemass D., 1987, MNRAS 228, 759

Conlon E.S., Dufton P.L., Keenan F.P., McCausland R.J.H., 1991, MNRAS 248, 820

Cristiani S., Vio R., 1990, A\&A 227, 385

Dahlem M., Schartel N., 1999, XMM Users' Handbook, Issue 1.1, ESA, Noordwijk

Fioc M., Rocca-Volmerange B., 1997, A\&A 326, 950

Fordham J.L.A., Bone D.A., Oldfield M.K., Bellis J.G., 1992, in: Morgan B.L. (ed.) Proceedings of the 10th Symposium on Photoelectronic Image Devices, held at Imperial
College of Science Technology and Medicine, London, 2-6 September 1991, Inst. Phys. Conf. Ser. 121, 105

Francis P.J., Hewett P.C., Foltz C.B., Chaffee F.H., Weymann R.J., Morris S.L., 1991, ApJ 373, 465

Giallongo E., Trevese D., 1990, ApJ 353, 24

Gunn J.E., Stryker L.L., 1983, ApJS 52, 121

Heck A., Egret D., Jaschek M., Jaschek C., 1984, A\&AS 57, 213

Irwin M., McMahon R.G., Hazard C., 1991, in: Crampton D. (ed.) The Space Distribution of Quasars, ASP Conf. Ser. 21,117

Johnson H.L., 1955, Ann. d'Ap. 18, 292

Koester D., 1999 (private communication)

Kurucz R.L., 1992, in: Barbuy B. and Renzini A. (eds.) The stellar populations of galaxies, IAU Symp. 149. Dordrecht, Kluwer Acad. Pub., p. 225

Lenz D.D., Newberg H.J., Rosner R., Richards G.T., Stoughton C., 1998, ApJS 119, 121

Madau P., 1995, ApJ 441, 18

Mason K.O., Cropper M.S., Hunt R., Horner S., Priedhorsky W., Ho C., Córdova F.A., Jamar C., Antonello E., 1996, in: Siegmund O.H. and Gummin M.A. (eds.) EUV, X-ray and Gamma-ray instrumentation for astronomy VII, Proc. SPIE 2808, 438

Miller L., Mitchell P.S., 1988, in: Osmer P.S., Porter A.C., Green R.F., Foltz C.B. (eds.) Proceedings of a workshop on optical surveys for quasars, ASP Conf. Ser. 2, 114

Moehler S., Sweigart A.V., Heber U., Landsman W.B., Catelan M., 1999, in: Noels A. et al. (eds.) The Galactic Halo - from Globular Clusters to Field Stars, $35^{\text {th }}$ Liège Int. Astroph. Coll. (in press)

Møller P., Jakobsen P., 1990, A\&A 228, 299

Møller P., Warren S., 1991, in: Crampton D. (ed.) The Space Distribution of Quasars, ASP Conf. Ser. 21, 96

Moreau O., Reboul H., 1995, A\&AS 111, 169

Oke J.B., Korycansky D.G., 1982, ApJ 255, 11

Prévot M.L., Lequeux J., Maurice E., Prévot L., RoccaVolmerange B., 1984, A\&A 132, 389

Royer P., 1994, Recherche de Candidats Quasars par la Méthode d'Analyse Multicouleurs, Master Thesis, University of Liège

Royer P., Manfroid J., 1996, A\&AS 119, 569

Schmidt M., Hasinger G., Gunn J., Schneider D., Burg R., Giacconi R., Lehmann M., MacKenty J., Trumper J., Zamorani G., 1998, A\&A 329, 495

Sterken C., Manfroid J., 1992, Astronomical Photometry - A Guide. Kluwer Academic Publishers, Dordrecht

Stocke J.T., Morris S.L., Gioia I.M., Maccacaro T., Schild R., Wolter A., Fleming T.A., Henry J.P., 1991, ApJS 76, 813

Warren S.J., Hewett P.C., Osmer P.S., 1994, ApJ 421, 412

Wesemael F., Auer L.H., Van Horn H.M., Savedoff M.P., 1980, ApJS 43, 159

Zheng W., Kriss G.A., Telfer R.C., Grimes J.P., Davidsen A.F., 1997, ApJ 475, 469 (erratum: 1998, ApJ 492, 855)

Zuo L., Lu L., 1993, ApJ 418, 601 